\title{
Outcome of shorter treatment duration in non-cavitary nodular bronchiectatic Mycobacterium avium complex lung disease
}

\author{
Yea Eun Park ${ }^{1}$, Yong Pil Chong ${ }^{2}$, Ye-Jee Kim ${ }^{3}$, Ock-Hwa Kim ${ }^{1}$, Byoung Soo Kwon ${ }^{4}$, Tae Sun Shim ${ }^{1}$, \\ Kyung-Wook Jo ${ }^{1}$ \\ ${ }^{1}$ Division of Pulmonology and Critical Care Medicine, Department of Internal Medicine, ${ }^{2}$ Department of Infectious Diseases, University of Ulsan \\ College of Medicine, Asan Medical Center, Seoul, South Korea; ${ }^{3}$ Department of Clinical Epidemiology and Biostatistics, Asan Medical Center, \\ Seoul, South Korea; ${ }^{4}$ Division of Pulmonary and Critical Care Medicine, Department of Internal Medicine, Seoul National University Bundang \\ Hospital, Seoul National University College of Medicine, Seongnam, South Korea \\ Contributions: (I) Conception and design: YE Park, TS Shim, KW Jo; (II) Administrative support: TS Shim; (III) Provision of study materials or \\ patients: YE Park, YJ Kim; (IV) Collection and assembly of data: All authors; (V) Data analysis and interpretation: YE Park, YP Chong, YJ Kim, KW \\ Jo; (VI) Manuscript writing: All authors; (VII) Final approval of manuscript: All authors. \\ Correspondence to: Kyung-Wook Jo, MD, PhD. Division of Pulmonary and Critical Care Medicine, Department of Internal Medicine, University of \\ Ulsan College of Medicine, Asan Medical Center, 88 Olympic-ro 43-gil, Songpa-gu, Seoul 05505, South Korea. Email: heathcliff6800@hanmail.net.
}

\begin{abstract}
Background: The recommended treatment duration for non-cavitary nodular bronchiectatic (NC-NB) Mycobacterium avium complex (MAC) lung disease (LD) is at least 12 months after culture conversion, but evidence supporting this is limited. This study investigated whether treatment for less than 12 months after culture conversion is acceptable in terms of recurrence rate.

Methods: The study enrolled the patients diagnosed with NC-NB MAC LD between 2001 and 2014 at a tertiary referral center in South Korea who received the standard treatment for at least 9 months after culture conversion up to October 2018. The patients were divided into a shorter treatment group (9-11 months after culture conversion) and a standard treatment group ( $\geq 12$ months).

Results: Of the 228 patients enrolled, 59 (25.9\%) were treated for 9-11 months after culture conversion and 169 (74.1\%) for $\geq 12$ months. The mean treatment durations after culture conversion in the shorter and standard treatment groups were 11.1 and 13.8 months, respectively $(\mathrm{P}<0.001)$. During median follow-up durations after the completion of treatment of 56.5 and 55.9 months, respectively, the recurrence rates in the two groups were similar, at 39.0\% (23/59) and 36.7\% (62/169). There were also no significant differences between the groups in the 1-year and 3-year recurrence rates.

Conclusions: Post-conversion treatment shorter than the recommended duration may be adequate in terms of recurrence rate for patients with NC-NB MAC LD who receive the standard treatment for at least 9 months after culture conversion.
\end{abstract}

Keywords: Mycobacterium avium complex (MAC); recurrence; treatment duration; culture conversion

Submitted Jul 28, 2019. Accepted for publication Jan 08, 2020.

doi: $10.21037 /$ jtd.2020.01.39

View this article at: http://dx.doi.org/10.21037/jtd.2020.01.39

\section{Introduction}

The incidence and prevalence of nontuberculous mycobacteria (NTM) disease are increasing worldwide, including in South Korea $(1,2)$. NTM comprise a diverse group of organisms, of which Mycobacterium avium complex (MAC) is the most frequently encountered in South
Korea (2). Its most common clinical manifestation in adult patients is MAC lung disease (LD) (3).

Until recently, there were considered to be three radiological types of MAC LD: nodular bronchiectatic $(\mathrm{NB})$, fibrocavitary (FC), and unclassifiable (4). However, Koh et al. found that the treatment outcomes for a cavitary form of NB 
disease were similar to those for the FC type and therefore suggested that the NB type should be further divided into cavitary $\mathrm{NB}(\mathrm{CNB})$ and non-cavitary $\mathrm{NB}$ (NC-NB) (5). Immediate treatment is not recommended for patients with the NC-NB form because this type is often indolent and the treatment is associated with possible adverse events (6). Although the treatment outcome for NC-NB MAC LD is favorable (5), the recurrence rate after successful treatment is extremely high, at around 25-50\% (5,7-9).

The guidelines for MAC LD treatment recommend that a macrolide-based regimen should be continued for at least 12 months after the sputum culture converts to negative $(4,10,11)$. However, supporting evidence for this recommendation is limited (12). The goal of treatment for so long after culture conversion is to achieve a relapse-free cure. Nevertheless, substantial numbers of patients with NC-NB MAC LD experience recurrence after successful treatment, and it is therefore possible that a shorter treatment period than the recommended 12 months would result in a similar recurrence rate. As yet, this has not been investigated. The aim of this study, therefore, was to investigate whether treatment for $<12$ months after culture conversion is acceptable in terms of recurrence rate for patients with the NC-NB form of MAC LD.

\section{Methods}

\section{Patients}

This retrospective study examined data for patients at Asan Medical Center, a 2,700-bed referral hospital in Seoul, South Korea, who met the American Thoracic Society (ATS) criteria for MAC LD between 2001 and 2014. Of these, the 451 patients who were treated with any type of antibiotic for $\geq 9$ months (i.e., 270 days) after culture conversion up to October 2018 were selected for the analysis. The 9 months cut-off criterion was arbitrary, chosen because we thought that at least 9 months of treatment after culture conversion would be required to keep recurrence after cure within an acceptable range. Patients were excluded if their treatment was other than by the standard regimen, they had radiological types of MAC LD other than the NC-NB form, the follow-up data were insufficient, or their MAC isolates were resistant to clarithromycin. The medical records of the remaining patients were analyzed in November 2018 (Figure 1).

The study protocol was approved by the Institutional Review Board (RFB) of Asan Medical Center (IRB No. 2018-0986), which waived the requirement for informed consent because of the retrospective nature of the analysis.

\section{Patient groups}

The included patients were classified into two groups on the basis of treatment duration: the shorter duration group, who received treatment for $\geq 9$ and $<12$ months after culture conversion, and the standard duration group, who received treatment for the standard duration of $\geq 12$ months after culture conversion. Culture conversion was defined as at least three consecutive negative mycobacterial cultures of respiratory samples, each collected at intervals of at least 4 weeks, with the date of the first negative culture defined as the date of culture conversion (13).

\section{Treatment regimen and treatment outcome analysis}

The inclusion and exclusion criteria selected only patients who received the standard regimen; thus, the included patients received the same macrolide-based regimen of clarithromycin or azithromycin along with rifampin and ethambutol, prescribed according to the patient's weight (4). In some patients, the treatment regimen was changed after initiation because of an adverse reaction to rifampin or ethambutol. In such cases, we regarded the treatment regimen as standard if both rifampin and ethambutol were prescribed for $\geq 80 \%$ of the total duration of the treatment (14). The treatment could be reinforced with parenteral drugs, such as streptomycin or amikacin, as decided by the attending physician.

Previous studies of the treatment outcomes of MAC LD analyzed treatment success rates $(5,15)$. However, the aim of the present study was to compare the recurrence rates between the two groups (shorter vs. standard duration), so we did not compare the treatment success rates. Instead, we assessed whether the recurrence rate in the shorter duration group was acceptable during the follow-up period after the completion of treatment. We also compared the 1 - and 3 -year recurrence rates between the two groups.

Redevelopment was defined as a subsequent diagnosis of NTM LD after completion of the initial treatment, irrespective of the causative NTM organism (5). Recurrence was defined as redevelopment in which two or more positive cultures were obtained for the same MAC species (M. avium or M. intracellulare) as that treated in the initial $\operatorname{LD}(5,15,16)$.

\section{Radiologic evaluation and microbiological examination}

Radiographic abnormalities on chest computed tomography 
at the time of diagnosis were categorized as FC, CNB, NC$\mathrm{NB}$, or unclassifiable, as previously defined $(5,17)$. The extent of the disease was assessed according to how many of the six lung lobes were involved, considering the lingular segment to be a separate lobe (9).

Expectorated sputum or samples obtained from bronchoscopy were cultured in both solid medium (Ogawa medium; Korean Institute of Tuberculosis, South Korea) and liquid medium (BACTEC 960 Mycobacterial Growth Indicator Tube; Becton Dickinson, Sparks, MD, USA). Acid-fast bacilli (AFB) smears were prepared by ZiehlNeelsen staining. Positive liquid cultures and colonies on the solid medium were subjected to polymerase chain reaction assays using Seeplex TB detection (Seegen, Seoul, Korea) to differentiate between a $M$. tuberculosis complex and NTM. The species of NTM isolates were identified by polymerase chain reaction and restriction fragment length polymorphism methods based on the $r p o B$ gene, or by reverse blot hybridization assays of $r p o B(18,19)$. The MAC isolates were identified as one of two species: $M$. avium or $M$. intracellulare.

\section{Statistical analysis}

The data are presented as means ( \pm standard deviations) or medians with interquartile ranges (IQRs) for continuous variables, and numbers (\%) for categorical variables. The data were compared by Student's $t$-tests or Mann-Whitney $U$ tests for continuous variables and $\chi^{2}$ or Fisher's exact tests for categorical variables. All the tests were two-sided and $\mathrm{P}$ values less than 0.05 were considered statistically significant. The recurrence rates after treatment completion were compared between the two groups by the KaplanMeier method. Multivariate logistic analyses were used to calculate adjusted odds ratios (ORs) and $95 \%$ confidence intervals (CIs) for the 1- and 3-year recurrence rates. The variables used in the multivariate logistic analyses were selected as those with a statistical significance level of $<0.1$ in the univariate analysis, together with treatment duration. The statistical analysis was performed using SPSS software (version 24.0; IBM Corp., Armonk, NY, USA).

\section{Results}

\section{Study subjects}

The eligibility screening identified 228 patients with macrolide-susceptible NC-NB MAC LD that had been treated with the standard regimen for $\geq 9$ months after sputum culture conversion. The mean age of the patients was 60.3 years, and $68.9 \%$ were female. Daily therapy was administered to almost all the patients $(97.4 \%, 222 / 228)$. Of the 228 patients, 59 (25.9\%) received treatment for 9-11 months after culture conversion (the shorter duration group), and the remaining 169 (74.1\%) received treatment for $\geq 12$ months (the standard duration group; Figure 1). The mean treatment durations after culture conversion in the two groups were $11.1 \pm 0.8$ months and $13.8 \pm 1.7$ months, respectively $(\mathrm{P}<0.001)$. The main reasons for stopping treatment earlier than the recommended 12 months were the attending physician's decision $(\mathrm{n}=55,93.2 \%)$, and adverse drug events $(n=4,6.8 \%)$. The two groups were comparable except that the shorter duration group was significantly more likely to have chronic liver disease as a comorbidity (Table 1).

\section{Recurrence rates after the completion of treatment}

The median (IQR) follow-up durations after the completion of treatment were 56.1 (31.3-78.1) months overall and 56.5 (31.8-83.6) and 55.9 (31.2-75.9) months for the shorter and standard duration groups, respectively $(\mathrm{P}=0.583)$ (Table 1). Of the 228 patients, 111 (48.7\%) experienced the redevelopment of NTM LD during follow-up, of which 85 (37.3\%) were recurrences. The recurrence rates in the two groups were similar: $39.0 \%(23 / 59)$ in the shorter duration group and $36.7 \%(62 / 169)$ in the standard duration group $(\mathrm{P}=0.753)$. The cumulative rates of recurrence in the two treatment groups based on the Kaplan-Meier method are shown in Figure 2; this showed no significant difference $(\mathrm{P}=0.713)$. Table 2 presents a comparison of clinical characteristics between the patients who experienced recurrence during the follow-up and those who did not. A similar proportion of each were in the shorter duration group (27.1\% vs. $25.2 \% ; \mathrm{P}=0.753$; Table 2).

\section{1- and 3-year recurrence rates}

Of the 228 patients, $210(92.1 \%)$ were followed up for at least a year after the completion of treatment. Of these, 53 received the shorter duration of treatment and 157 received the standard duration. Recurrence occurred in $17.0 \%(9 / 53)$ of the shorter duration patients and $12.7 \%(20 / 157)$ of the standard duration patients $(\mathrm{P}=0.439)$. Table 3 compares the characteristics of the patients according to whether they experienced recurrence in the first year. A significantly 
451 patients who met the ATS criteria for MAC lung disease between 2001 and 2014, treated with any type antibiotics for $\geq 9$ months after culture conversion until October 2018

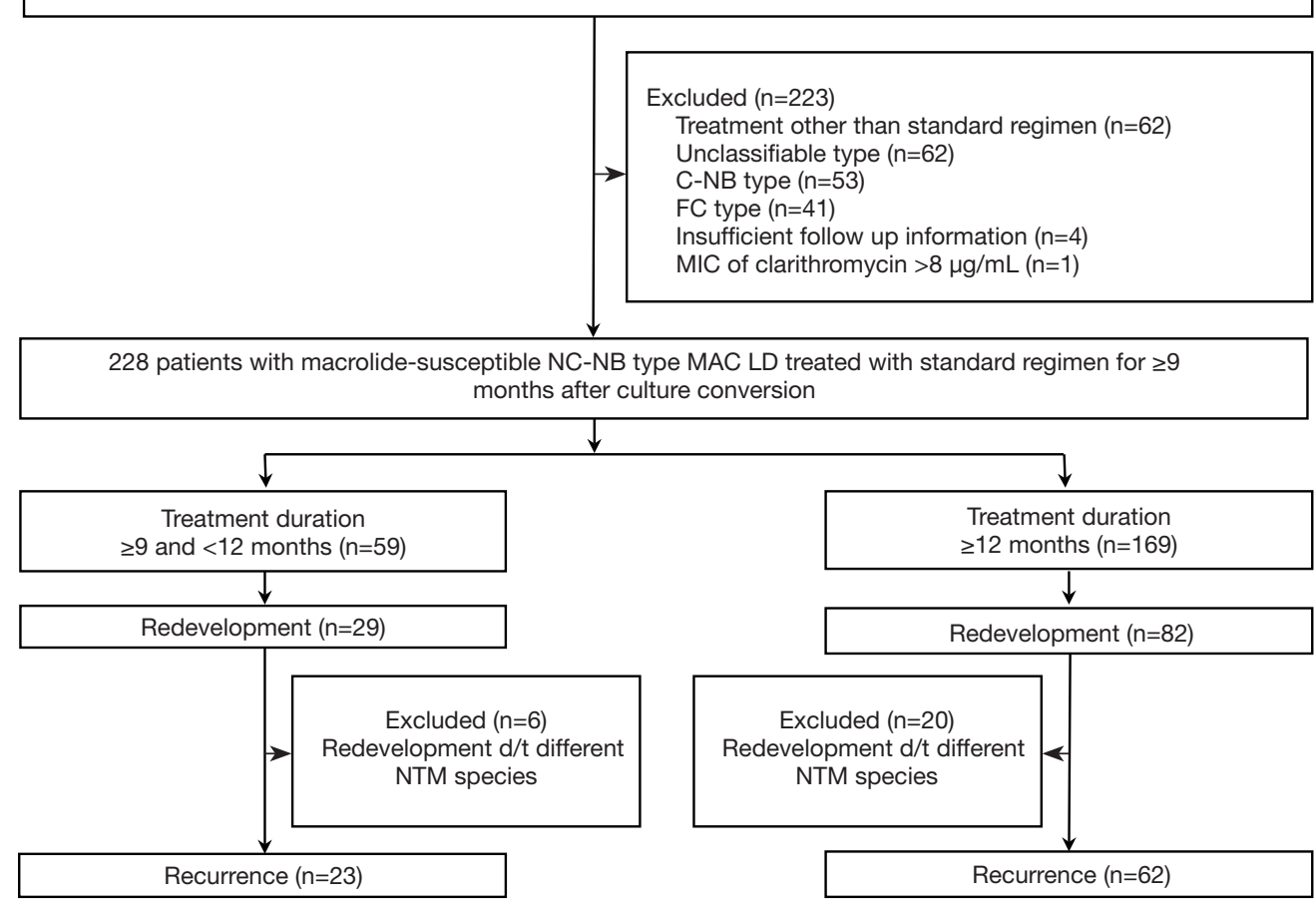

Figure 1 Study flow chart. ATS, American Thoracic Society; MAC, Mycobacterium avium complex; CNB, cavitary nodular bronchiectatic; FC, fibrocavitary; MIC, minimum inhibitory concentration; NC-NB, non-cavitary nodular bronchiectatic; MAC LD, Mycobacterium avium complex lung disease; NTM, nontuberculous mycobacteria.

higher proportion of those with 1-year recurrence were aged $\geq 60$ years.

Of the 163 patients followed up for at least three years after the end of treatment, 43 were in the shorter duration group and 120 in the standard duration group. Recurrence occurred in $41.9 \%(18 / 43)$ and $36.7 \%(44 / 120)$ of these groups, respectively $(\mathrm{P}=0.547)$. Table 4 compares the characteristics of the patients who experienced recurrence during the three years and those who did not. Most clinical characteristics, including treatment duration after culture conversion, were comparable in the two groups, although there were significant differences in the proportions of those aged $\geq 60$ years and with a previous history of NTM treatment, and in the number of involved lobes.

\section{Multivariate analysis}

Table 5 summarizes the results of the multivariate analysis, which revealed that age $\geq 60$ years was the only significant risk factor for 1 - and 3-year recurrence after treatment completion (1-year recurrence: adjusted OR, 2.565; 95\%
CI, 1.020-6.450; $\mathrm{P}=0.045$; 3-year recurrence: adjusted $\mathrm{OR}$, 2.511; 95\% CI, 1.256-5.019; $\mathrm{P}=0.009$ ). Neither the 1-year nor the 3-year recurrence rates were significantly associated with the length of treatment after culture conversion in the multivariate analyses.

\section{Discussion}

Although the guidelines recommend that macrolide-based regimens for treating MAC LD should be administered for at least 12 months after sputum culture conversion $(4,10,11)$, the supporting evidence for this is limited $(12,20)$. A substantial proportion of patients with the NC-NB form of MAC LD eventually experience recurrence after successful treatment; this suggested that a shorter duration of treatment might yield similar results in terms of recurrence rate. To the best of our knowledge this is the first study to investigate whether this was the case. The study was conducted in a single tertiary referral center in South Korea through a retrospective analysis of consecutive patients with MAC LD, and its most important finding was that 
Table 1 Baseline characteristics and treatment outcomes of 228 patients with macrolide-susceptible non-cavitary nodular bronchiectatic Mycobacterium avium complex lung disease, compared between those with shorter treatment duration (9-11 months) and standard duration ( $\geq 12$ months)

\begin{tabular}{|c|c|c|c|c|}
\hline Characteristic/outcome & Total $(n=228)$ & Shorter duration $(n=59)$ & Standard duration $(n=169)$ & $\mathrm{P}$ \\
\hline Age $\geq 60$ years & $123(53.9)$ & 35 (59.3) & $88(52.1)$ & 0.336 \\
\hline Female & $157(68.9)$ & $39(66.1)$ & $118(69.8)$ & 0.595 \\
\hline BMI $\left(\mathrm{kg} / \mathrm{m}^{2}\right)$ & $20.6 \pm 2.6$ & $20.7 \pm 2.8$ & $20.6 \pm 2.6$ & 0.919 \\
\hline Previous history of TB treatment & 75 (32.9) & $22(37.3)$ & $53(31.4)$ & 0.404 \\
\hline Previous history of NTM treatment & $14(6.1)$ & $6(10.2)$ & $8(4.7)$ & 0.134 \\
\hline \multicolumn{5}{|l|}{ Comorbidity } \\
\hline COPD & $26(11.4)$ & $9(15.3)$ & $17(10.1)$ & 0.280 \\
\hline Malignancy & $43(18.9)$ & $11(18.6)$ & $32(18.9)$ & 0.961 \\
\hline Etiology & & & & 0.410 \\
\hline Mycobacterium avium & $111(48.7)$ & $26(44.1)$ & $85(50.3)$ & \\
\hline Mycobacterium intracellulare & $117(51.3)$ & $33(55.9)$ & $84(49.7)$ & \\
\hline Positive AFB smear at treatment initiation & $77(33.8)$ & $24(40.7)$ & $53(31.4)$ & 0.193 \\
\hline Number of involved lobes & $3.5 \pm 1.3$ & $3.5 \pm 1.3$ & $3.4 \pm 1.4$ & 0.380 \\
\hline Use of injectable aminoglycosides & $87(38.2)$ & $21(35.6)$ & $66(39.1)$ & 0.638 \\
\hline Daily therapy & $222(97.4)$ & $58(98.3)$ & $164(97.0)$ & $>0.999$ \\
\hline
\end{tabular}

BMI, body mass index; TB, tuberculosis; NTM, nontuberculous mycobacteria; COPD, chronic obstructive lung disease; AFB, acid-fast bacilli. Data are presented as mean ( \pm standard deviation), median (interquartile range), or number (\%).

the recurrence rate after treatment completion was similar between the shorter treatment group and the standard treatment group. Thus, treatment for a shorter period than the recommended 12 months after culture conversion may be acceptable for patients with NC-NB MAC LD who have received the standard treatment for at least 9 months after culture conversion.

The recommendation to maintain treatment for at least 12 months after culture conversion in MAC LD is based on only limited evidence $(12,20)$. In one study $(12)$, Wallace et al. reported intermediate results for 50 patients with
MAC LD treated with clarithromycin regimens between January 1992 and October 1994, concluding that "patients should be treated until culture-negative 10 to 12 months." This was based on their finding that none of the 23 patients who had maintained culture negativity for a mean of 12.0 months while receiving therapy experienced recurrence during a mean of 19.1 months follow-up after treatment completion. In 2002, another study reported that new positive sputum cultures after culture negativity for 10 to 12 months treatment were usually due to reinfection rather than disease relapse, supporting the recommendation for 


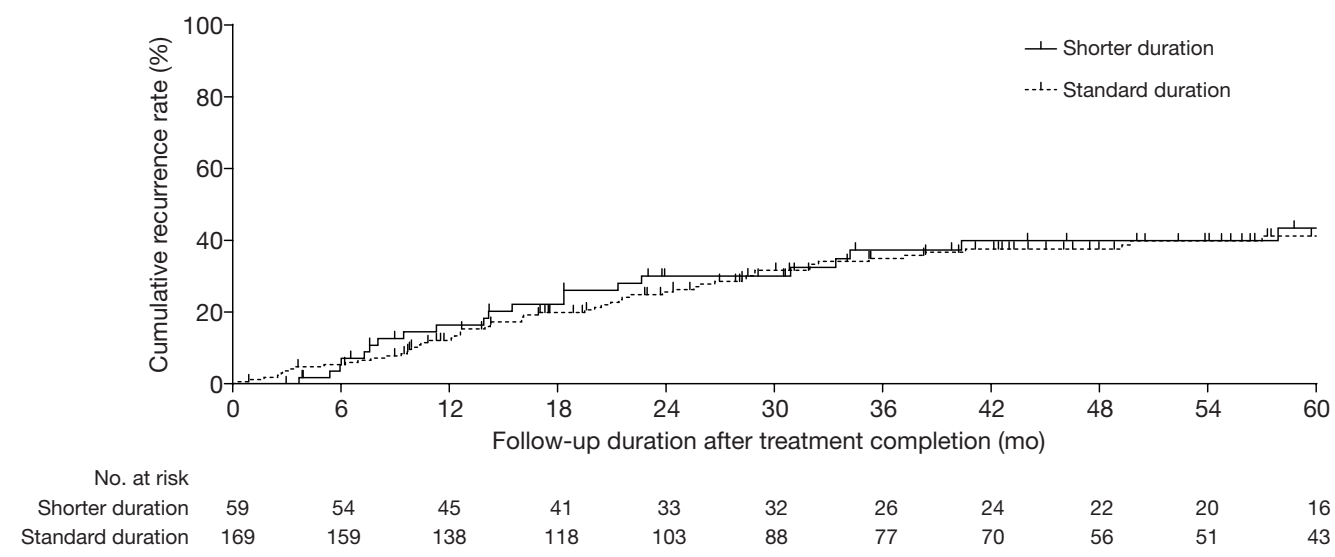

Figure 2 Kaplan-Meier estimates of the cumulative recurrence rates after treatment completion according to the duration of treatment after culture conversion: shorter duration (9-11 months) or standard duration ( $\geq 12$ months). The recurrence rates in the two groups were similar ( $\mathrm{P}=0.713$ by the Gehan-Breslow test).

Table 2 Clinical characteristics of the 228 patients, compared according to whether there was recurrence during follow-up

\begin{tabular}{|c|c|c|c|c|}
\hline Characteristic & Total $(n=228)$ & Recurrence $(n=85)$ & No recurrence $(n=143)$ & $\mathrm{P}$ \\
\hline Age (y) & $60.3 \pm 10.7$ & $61.2 \pm 10.1$ & $59.7 \pm 11.0$ & 0.329 \\
\hline Age $\geq 60$ years & $123(53.9)$ & $53(62.4)$ & $70(49.0)$ & 0.050 \\
\hline $\mathrm{BMI}\left(\mathrm{kg} / \mathrm{m}^{2}\right)$ & $20.6 \pm 2.6$ & $20.7 \pm 2.3$ & $20.6 \pm 2.8$ & 0.784 \\
\hline Current or past smoker & $43(18.9)$ & $15(17.6)$ & $28(19.6)$ & 0.718 \\
\hline Previous history of NTM treatment & $14(6.1)$ & $9(10.6)$ & $5(3.5)$ & 0.031 \\
\hline \multicolumn{5}{|l|}{ Comorbidity } \\
\hline COPD & $26(11.4)$ & $10(11.8)$ & $16(11.2)$ & 0.895 \\
\hline Diabetes mellitus & $17(7.5)$ & $10(11.8)$ & $7(4.9)$ & 0.056 \\
\hline Mycobacterium avium & $111(48.7)$ & $40(47.1)$ & $71(49.7)$ & \\
\hline Mycobacterium intracellulare & $117(51.3)$ & $45(52.9)$ & $72(50.3)$ & \\
\hline Positive AFB smear at treatment initiation & $77(33.8)$ & $30(35.3)$ & $47(32.9)$ & 0.708 \\
\hline Number of involved lobes & $3.5 \pm 1.3$ & $3.7 \pm 1.3$ & $3.4 \pm 1.3$ & 0.079 \\
\hline Use of injectable aminoglycosides & $87(38.2)$ & $28(32.9)$ & $59(41.3)$ & 0.211 \\
\hline Daily therapy & $222(97.4)$ & $85(100.0)$ & $137(95.8)$ & 0.087 \\
\hline Surgical resection & $5(2.2)$ & $3(3.5)$ & $2(1.4)$ & 0.364 \\
\hline Shorter treatment duration (9-11 months) & $59(25.9)$ & $23(27.1)$ & $36(25.2)$ & 0.753 \\
\hline
\end{tabular}

BMI, body mass index; TB, tuberculosis; NTM, nontuberculous mycobacteria; COPD, chronic obstructive lung disease; AFB, acid-fast bacilli. Data are presented as mean ( \pm standard deviation), median (interquartile range), or number (\%). 
Table 3 Clinical characteristics of the 210 patients followed up for at least 1 year, compared according to whether or not they experienced recurrence

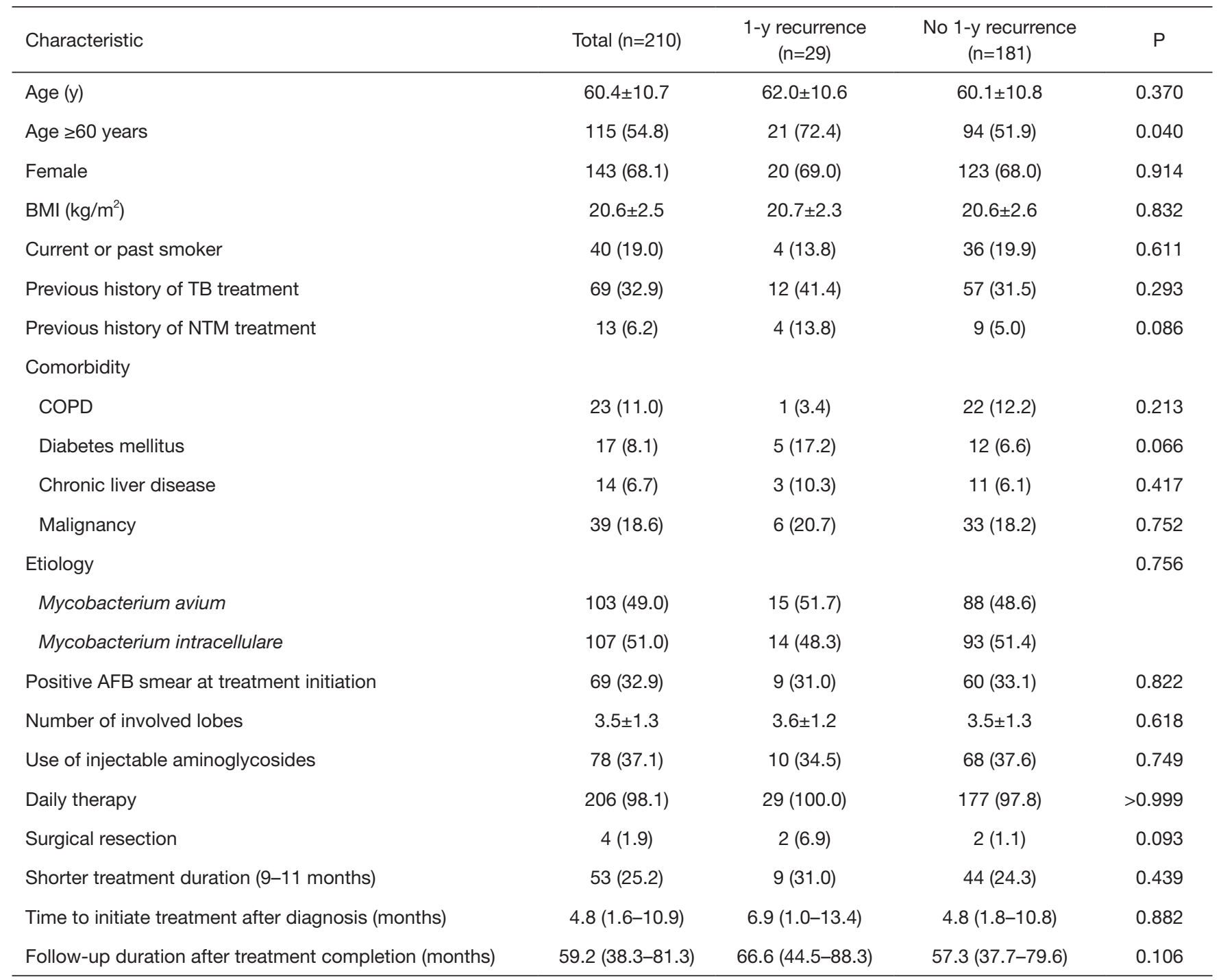

BMI, body mass index; TB, tuberculosis; NTM, nontuberculous mycobacteria; COPD, chronic obstructive lung disease; AFB, acid-fast bacilli. Data are presented as mean ( \pm standard deviation), median (interquartile range), or number (\%).

12 months treatment after culture conversion (20). These studies have provided the evidence base for guidelines including those of the ATS in 2007 (4) and of the British Thoracic Society guidelines in 2017 (10), as well as for expert opinion $(1,11,21)$. However, no further study since these guidelines were published has investigated whether the duration of MAC LD therapy should be 12 months beyond sputum culture conversion. In the present study we showed that, at least for the NC-NB form of MAC $\mathrm{LD}$, a treatment duration shorter than the recommended 12 months after culture conversion may be adequate.
In our study, the mean duration of the treatment of the shorter treatment group was nearly 3 months shorter than that of the standard group. Although the mean treatment duration for the shorter treatment group was 11.1 months, which may appear to differ only to a small extent from the recommended "at least 12 months" of treatment, in practice many attending physicians treat patients for far longer than 12 months as a result of the words "at least". Treatment durations after culture conversion of around 16-18 months have been reported in previous studies $(5,9,16,22)$. In the present study, the mean treatment duration in the standard 
Table 4 Clinical characteristics of the 163 patients followed up for at least 3 years, compared according to whether or not they experienced recurrence

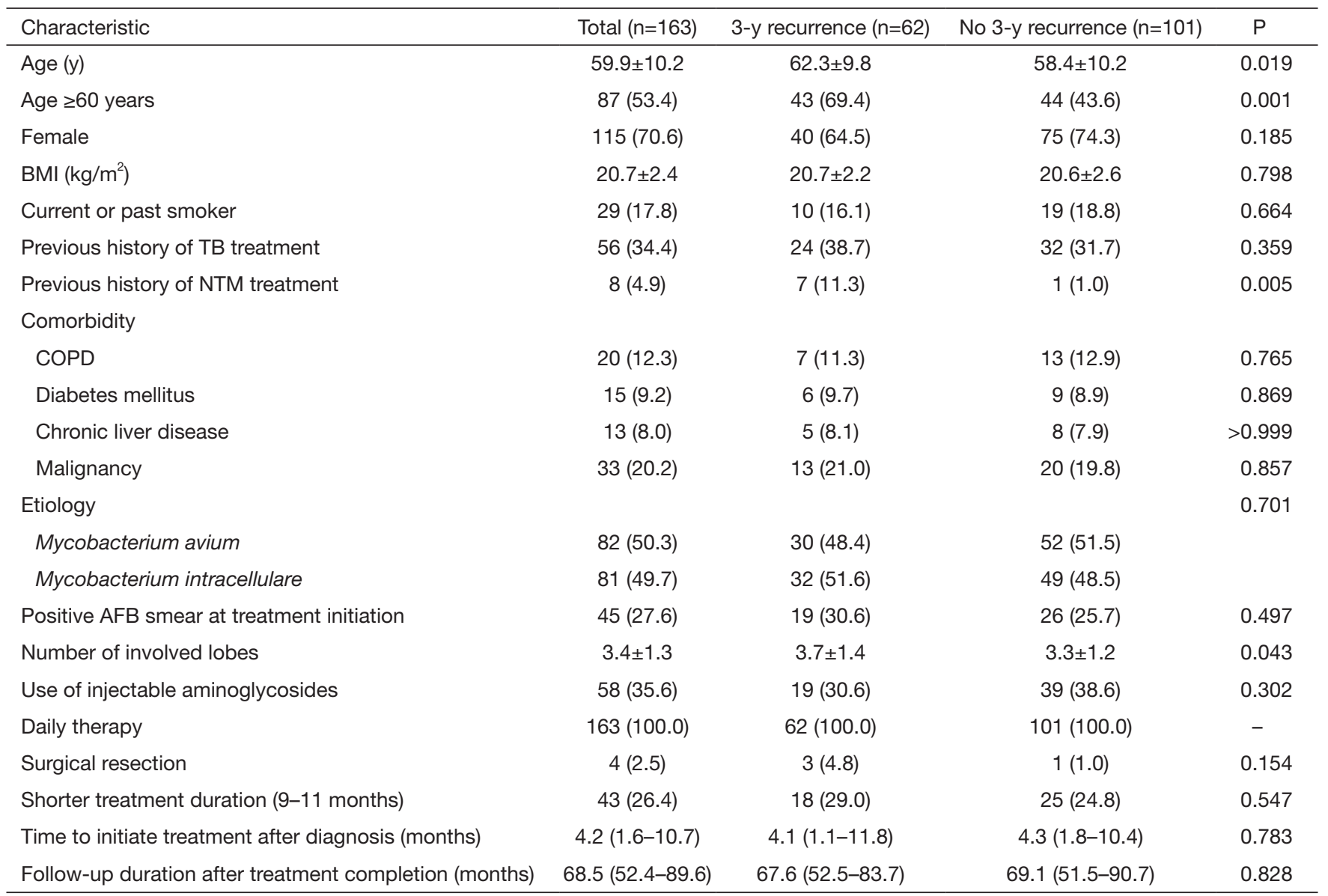

BMI, body mass index; TB, tuberculosis; NTM, nontuberculous mycobacteria; COPD, chronic obstructive lung disease; AFB, acid-fast bacilli. Data are presented as mean ( \pm standard deviation), median (interquartile range), or number $(\%)$.

Table 5 Analyses of factors affecting recurrence in non-cavitary nodular bronchiectatic Mycobacterium avium complex lung disease

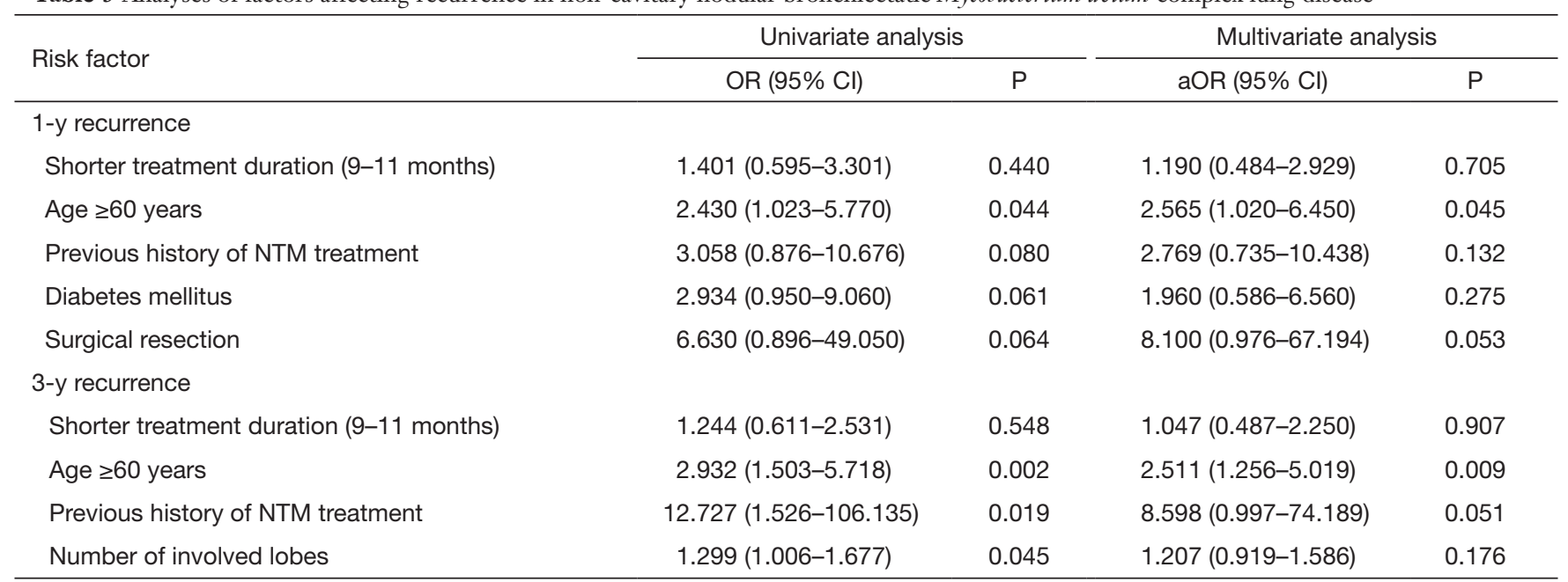

OR, odds ratio; $\mathrm{Cl}$, confidence interval; aOR, adjusted OR; NTM, nontuberculous mycobacteria lung disease. 
treatment group was approximately 14 months. Given the relatively long treatment period after culture conversion in clinical practice, reducing the treatment duration to 11 months would be a meaningful difference. Considering the well-known adverse events associated with long-term antimycobacterial treatments (23) and the costs associated with the treatment, we believe that a shorter treatment duration might be acceptable, at least for a subset of patients. It should be emphasized that we do not believe treatment for a shorter duration would be applicable to all patients with the NC-NB form of MAC LD. Stopping treatment earlier than recommended can potentially lead to the incomplete eradication of the causative organism, resulting in the eventual recurrence of the original strain. This treatment strategy should therefore be employed with caution for selected patients, such as those with an initially low mycobacterial burden who experience adverse drug events and whose treatment duration was close to the recommended length. A prospective randomized trial is needed to establish which patients could be treated for a shorter duration.

Recurrence after successful treatment can be the result of a relapse of the same MAC strain or reinfection with a new strain. The period between the end of treatment and recurrence is shorter with a relapse than with reinfection: a true relapse generally occurs within 1 year, whereas reinfection typically occurs after $1-2$ years $(5,9,16,22)$. Reinfection after treatment success is due to exposure to an environmental source, such as water or soil (24); household tap water tends to be the main source of $M$. avium pulmonary infection, whereas $M$. intracellulare pulmonary infections are generally acquired from soil (25). This is a particular issue for individuals with immune deficiencies, which predispose them to pulmonary MAC infections (26). In our study, the 1-year recurrence rate was slightly higher in the shorter duration group, although the difference was not statistically significant. Considering that recurrence in the first year is more likely to be due to a relapse, the residual mycobacterial burden of the initial strain could be responsible for this slight difference in recurrence rate in the shorter treatment group. However, the difference in recurrence rate between the two groups seemed to disappear with longer follow-up, as shown in Figure 2. The 3-year recurrence rates, which are more likely to reflect reinfection by a different strain, were similar. These findings suggest that the shorter treatment duration eventually resulted in a similar recurrence rate to that with the standard duration, with a possible slight increase in relapse rate soon after the end of treatment, but ultimately a similar reinfection rate as time elapsed. However, we did not genotype the isolates, so we were not able to determine whether these recurrences were due to relapse or reinfection.

Current guidelines recommend the same treatment duration after culture conversion for all subtypes of MAC LD irrespective of the presence of cavitation (i.e., FC or CNB vs. NC-NB). Cavitation is the classic hallmark of a very high mycobacterial burden in mycobacterial LD (27). Thus, there are more bacilli in the lungs in cavitary MAC LD. In addition, the destruction of the vascular architecture of the cavity limits drug supply and penetration into the cavitary lesion (28), so a longer duration of total treatment might be needed in cavitary MAC LD. It is also possible that the remnant mycobacterial burden after culture conversion is higher in MAC LD with cavitation, requiring a longer treatment duration after microbiologic conversion to eradicate the remnant organisms. This could be another reason for considering shorter treatments for the NC-NB form of MAC LD than for the FC or CNB forms.

In the present study, age $\geq 60$ years was a statistically significant risk factor for both 1 - and 3-year recurrence. There are several plausible explanations for this. First, there are various host immune responses to MAC infection, such as the secretion of interferon gamma, tumor necrosis factor alpha, interleukin-6, interleukin-12, and TH1 immunity triggered through Toll-like receptors (29). Waning immunity with age could be one reason why older patients might be more susceptible with reinfection with a MAC organism after the successful eradication of the initial infection. Second, approximately $90 \%$ of South Koreans have received the bacillus Calmette-Guérin (BCG) vaccination, which is mandatory because of the high incidence of tuberculosis in South Korea (30). In addition, before 1997, those with the negative tuberculin skin test at age 12 years were revaccinated with BCG. The BCG vaccination is known to be protective against infection with M. avium (31). However, a recent study has reported that the vaccine effectiveness of BCG seemed to wane beyond 20 years after vaccination (32). Thus, the decreased effectiveness of the BCG vaccination may be another reason for the higher recurrence rate due to reinfection of a MAC organism among the patients aged $\geq 60$ years, whose BCG vaccination was administered at least 40 years previously.

Our study had several limitations. Most significantly, our analysis was conducted at a single referral center, with a non-randomized retrospective design. In addition, the decision about treatment duration was mainly made by the attending physician, so it is likely to have been determined 
by unmeasured factors that affected the patients' compliance with treatment. We were unable to identify the exact reason for the decision for a shorter treatment duration for most of the 59 patients. It is uncertain whether the results of this study can be applied to those undergoing intermittent therapy, as most of our patients received daily therapy. Finally, we did not genotype the isolates, so we were unable to distinguish between relapse and reinfection.

In conclusion, during a median follow-up of approximately 5 years, the recurrence rate in patients with NC-NB MAC LD who received the standard treatment for 9-11 months after achieving culture conversion was similar to that of patients who received treatment for the recommended $\geq 12$ months. This finding suggests that postconversion treatment that is shorter than the recommended duration may be applicable for selected patients with NCNB MAC LD.

\section{Acknowledgments}

Funding: This work was supported by the National Research Foundation of Korea grant funded by the Korea government (Ministry of Science and ICT) (No. 2019R1F1A1059190).

\section{Footnote}

Conflicts of Interest: The authors have no conflicts of interest to declare.

Ethical Statement: The authors are accountable for all aspects of the work in ensuring that questions related to the accuracy or integrity of any part of the work are appropriately investigated and resolved. The study protocol was approved by the Institutional Review Board (RFB) of Asan Medical Center (IRB No. 2018-0986), which waived the requirement for informed consent because of the retrospective nature of the analysis.

Open Access Statement: This is an Open Access article distributed in accordance with the Creative Commons Attribution-NonCommercial-NoDerivs 4.0 International License (CC BY-NC-ND 4.0), which permits the noncommercial replication and distribution of the article with the strict proviso that no changes or edits are made and the original work is properly cited (including links to both the formal publication through the relevant DOI and the license). See: https://creativecommons.org/licenses/by-nc-nd/4.0/.

\section{References}

1. Kwon YS, Koh WJ. Diagnosis and Treatment of Nontuberculous Mycobacterial Lung Disease. J Korean Med Sci 2016;31:649-59.

2. Ko RE, Moon SM, Ahn S, et al. Changing Epidemiology of Nontuberculous Mycobacterial Lung Diseases in a Tertiary Referral Hospital in Korea between 2001 and 2015. J Korean Med Sci 2018;33:e65.

3. Ryu YJ, Koh WJ, Daley CL. Diagnosis and Treatment of Nontuberculous Mycobacterial Lung Disease: Clinicians' Perspectives. Tuberc Respir Dis (Seoul) 2016;79:74-84.

4. Griffith DE, Aksamit T, Brown-Elliott BA, et al. An official ATS/IDSA statement: diagnosis, treatment, and prevention of nontuberculous mycobacterial diseases. Am J Respir Crit Care Med 2007;175:367-416.

5. Koh WJ, Moon SM, Kim SY, et al. Outcomes of Mycobacterium avium complex lung disease based on clinical phenotype. Eur Respir J 2017;50. doi: 10.1183/13993003.02503-2016.

6. Lande L, George J, Plush T. Mycobacterium avium complex pulmonary disease: new epidemiology and management concepts. Curr Opin Infect Dis 2018;31:199-207.

7. Boyle DP, Zembower TR, Reddy S, et al. Comparison of Clinical Features, Virulence, and Relapse among Mycobacterium avium Complex Species. Am J Respir Crit Care Med 2015;191:1310-7.

8. Kwon BS, Shim TS, Jo KW. The second recurrence of Mycobacterium avium complex lung disease after successful treatment for first recurrence. Eur Respir J 2019;53. doi: 10.1183/13993003.01038-2018.

9. Lee BY, Kim S, Hong Y, et al. Risk factors for recurrence after successful treatment of Mycobacterium avium complex lung disease. Antimicrob Agents Chemother 2015;59:2972-7.

10. Haworth CS, Banks J, Capstick T, et al. British Thoracic Society guidelines for the management of non-tuberculous mycobacterial pulmonary disease (NTM-PD). Thorax 2017;72:ii1-64.

11. Kwon YS, Koh WJ, Daley CL. Treatment of Mycobacterium avium Complex Pulmonary Disease. Tuberc Respir Dis (Seoul) 2019;82:15-26.

12. Wallace RJ Jr, Brown BA, Griffith DE, et al. Clarithromycin regimens for pulmonary Mycobacterium avium complex. The first 50 patients. Am J Respir Crit Care Med 1996;153:1766-72.

13. van Ingen J, Aksamit T, Andrejak C, et al. Treatment 
outcome definitions in nontuberculous mycobacterial pulmonary disease: an NTM-NET consensus statement. Eur Respir J 2018;51.

14. Kwon BS, Kim MN, Sung H, et al. In Vitro MIC Values of Rifampin and Ethambutol and Treatment Outcome in Mycobacterium avium Complex Lung Disease. Antimicrob Agents Chemother 2018;62:e00491-18.

15. Kim OH, Kwon BS, Han M, et al. Association between duration of aminoglycoside treatment and outcome of cavitary Mycobacterium avium complex lung disease. Clin Infect Dis 2019;68:1870-6.

16. Wallace RJ Jr, Brown-Elliott BA, McNulty S, et al. Macrolide/Azalide therapy for nodular/bronchiectatic mycobacterium avium complex lung disease. Chest 2014;146:276-82.

17. Hwang JA, Kim S, Jo KW, et al. Natural history of Mycobacterium avium complex lung disease in untreated patients with stable course. Eur Respir J 2017;49. pii: 1600537.

18. Lee H, Park HJ, Cho SN, et al. Species identification of mycobacteria by PCR-restriction fragment length polymorphism of the rpoB gene. J Clin Microbiol 2000;38:2966-71.

19. Sanguinetti M, Posteraro B, Ardito F, et al. Routine Use of PCR-Reverse Cross-Blot Hybridization Assay for Rapid Identification of Mycobacterium Species Growing in Liquid Media. J Clin Microbiol 1998;36:1530-3.

20. Wallace RJ Jr, Zhang Y, Brown-Elliott BA, et al. Repeat positive cultures in Mycobacterium intracellulare lung disease after macrolide therapy represent new infections in patients with nodular bronchiectasis. J Infect Dis 2002;186:266-73.

21. Stout JE, Koh WJ, Yew WW. Update on pulmonary disease due to non-tuberculous mycobacteria. Int J Infect Dis 2016;45:123-34.

22. Boyle DP, Zembower TR, Qi C. Relapse versus Reinfection of Mycobacterium avium Complex Pulmonary Disease. Patient Characteristics and Macrolide Susceptibility. Ann Am Thorac Soc 2016;13:1956-61.

Cite this article as: Park YE, Chong YP, Kim YJ, Kim OH, Kwon BS, Shim TS, Jo KW. Outcome of shorter treatment duration in non-cavitary nodular bronchiectatic Mycobacterium avium complex lung disease. J Thorac Dis 2020;12(3):338-348. doi: $10.21037 /$ jtd.2020.01.39
23. Kamii Y, Nagai H, Kawashima M, et al. Adverse reactions associated with long-term drug administration in Mycobacterium avium complex lung disease. Int J Tuberc Lung Dis 2018;22:1505-10.

24. Nishiuchi Y, Iwamoto T, Maruyama F. Infection Sources of a Common Non-tuberculous Mycobacterial Pathogen, Mycobacterium avium Complex. Front Med (Lausanne) 2017;4:27.

25. Wallace RJ Jr, Iakhiaeva E, Williams MD, et al. Absence of Mycobacterium intracellulare and presence of Mycobacterium chimaera in household water and biofilm samples of patients in the United States with Mycobacterium avium complex respiratory disease. J Clin Microbiol 2013;51:1747-52.

26. Wu UI, Holland SM. Host susceptibility to nontuberculous mycobacterial infections. Lancet Infect Dis 2015;15:968-80.

27. Ong CW, Elkington PT, Friedland JS. Tuberculosis, pulmonary cavitation, and matrix metalloproteinases. Am J Respir Crit Care Med 2014;190:9-18.

28. Dartois V. The path of anti-tuberculosis drugs: from blood to lesions to mycobacterial cells. Nat Rev Microbiol 2014;12:159-67.

29. Orme IM, Ordway DJ. Host response to nontuberculous mycobacterial infections of current clinical importance. Infect Immun 2014;82:3516-22.

30. Hong YP, Kim SJ, Lew WJ, et al. The seventh nationwide tuberculosis prevalence survey in Korea, 1995. Int J Tuberc Lung Dis 1998;2:27-36.

31. Orme IM, Collins FM. Efficacy of Mycobacterium bovis BCG vaccination in mice undergoing prior pulmonary infection with atypical mycobacteria. Infect Immun 1984;44:28-32.

32. Nguipdop-Djomo P, Heldal E, Rodrigues LC, et al. Duration of BCG protection against tuberculosis and change in effectiveness with time since vaccination in Norway: a retrospective population-based cohort study. Lancet Infect Dis 2016;16:219-26. 